\title{
Effects of targeted non-technical skills training on recognition and treatment of cardiac arrest
}

Medical University of Graz

Purpose of the study

The aim of the study was to investigate effects of targeted non-technical skills (NTS) training on recognition of cardiac arrest, timing of cardiopulmonary resuscitation (CPR) and timing of defibrillation.

\section{Materials and methods}

A multi-center-controlled trial was performed within ERC Immediate Life Support (ILS) course centers in Austria from June 2013 to September 2014. The control group (CG) received standard ILS training according to course rules. The intervention group $(I G)$ received an additional 1-hour interactive and video-supported, targeted NTS training. Equally predefined cardiac arrest simulations (CAS) according to the ILS course documents were video-recorded in both groups with previous informed consent. Investigated parameters included the onset time of recognition of cardiac arrest until CPR start (OT-to-CPR) and the onset time until completion of first rhythm check (OT-to-RC).

\section{Results}

30 ILS courses were included, whereas 18 were allocated as CG and 12 as IG. 48 CAS were recorded and evaluated (IG: $n=25 ; C G: n=23)$. OT-to-CPR was highly significantly lower in the IG $(p=0.009 ; \mid G M=20.96 s, S D=8,10 s ; C G$ $M=33.00$ s, $S D=18.52 \mathrm{~s})$. OT-to-RC was significantly lower in the IG $(p=0.012 ; \mid G M=64.56 s, S D=14,61 s$; $C G$ $M=80.09 \mathrm{~s}, \mathrm{SD}=25,65 \mathrm{~s})$.
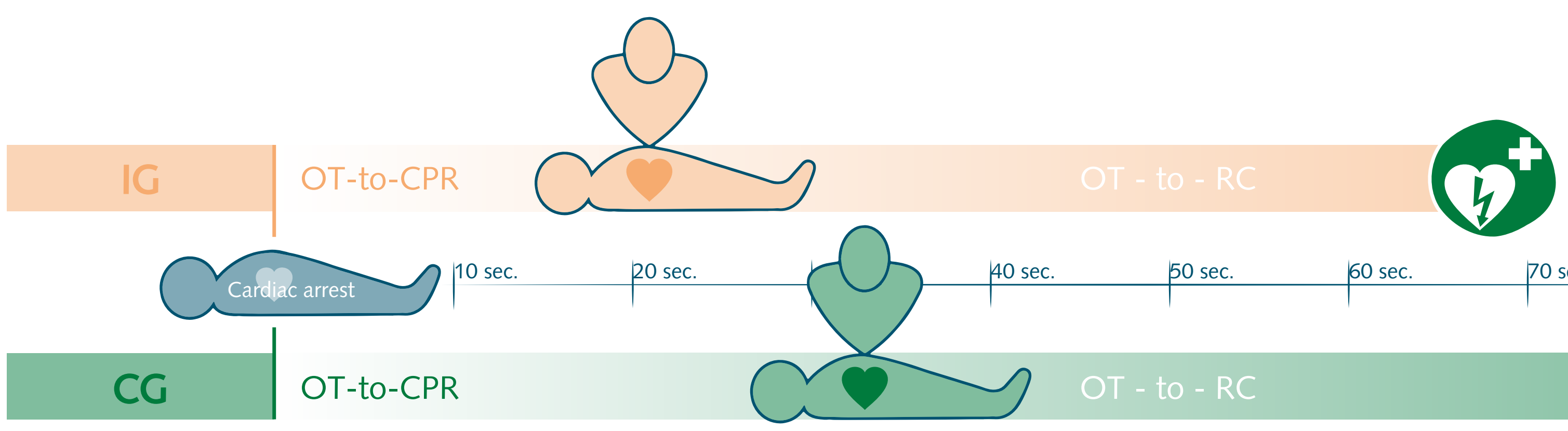

40 sec.
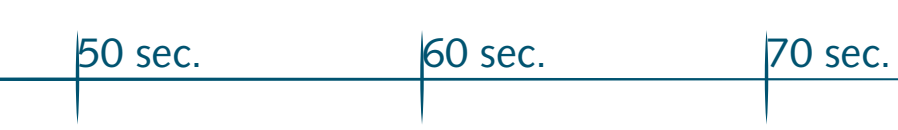
80

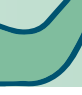

David Jäger ${ }^{1}$, Georg Prattes ${ }^{1}$

Lucas Pflanzl-Knizacek², Joachim Schlieber ${ }^{3}$, Gerhard Prause ${ }^{1}$

Division of General Anaesthesiology, Emergency- and Intensive Care Medicine Medical University of Graz, Austria

Division of Endocrinology and Diabetology, Medical University of Graz, Austria

${ }^{3}$ AUVA Trauma Centre Salzburg, Austria

Contact: georg.prattes@medunigraz.at

Conclusions

Our results proof that there are significant effects of targeted NTS training on recognition and treatment of cardiac arrest. The duration of NTS training shows that a relatively short amount of training is able to significantly improve team performance. Possibly leading to earlier CPR and defibrillation, targeted NTS training can be recommended as additional training in life support courses. 